Meta

Journal des traducteurs

Translators' Journal

\title{
The many ways of saying "pattern" in French medical texts
}

\section{Karin R. M. Band}

Volume 46, numéro 1, mars 2001

Traduction médicale et documentation / Medical translation and documentation

URI : https://id.erudit.org/iderudit/001965ar

DOI : https://doi.org/10.7202/001965ar

Aller au sommaire du numéro

Éditeur(s)

Les Presses de l'Université de Montréal

ISSN

0026-0452 (imprimé)

1492-1421 (numérique)

Découvrir la revue

Citer cet article

Band, K. R. M. (2001). The many ways of saying "pattern” in French medical texts. Meta, 46(1), 22-33. https://doi.org/10.7202/001965ar

\section{Résumé de l'article}

En français, pattern est un anglicisme dont le sens et le domaine d'application sont sensiblement plus restreints que ceux du terme anglais. À quelques exceptions près, il n'y a pas de terme unique qui puisse être utilisé pour traduire pattern (le " mot manquant »). Par conséquent, les acceptions du terme anglais exigent des traductions différenciées. Le traducteur travaillant à partir du français doit reconnaître le mot pattern derrière les termes français, pour remettre ce « mot caché » là où il est requis dans la langue cible. L'article présente des cas d'espèce trouvés dans des sources françaises appariées pour le contexte.
Ce document est protégé par la loi sur le droit d'auteur. L’utilisation des services d’Érudit (y compris la reproduction) est assujettie à sa politique d'utilisation que vous pouvez consulter en ligne.

https://apropos.erudit.org/fr/usagers/politique-dutilisation/ 


\title{
The many ways of saying "pattern" in French medical texts
}

\author{
KARIN R. M. BAND \\ Medical linguist, London, United Kingdom
}

\begin{abstract}
RÉSUMÉ
En français, pattern est un anglicisme dont le sens et le domaine d'application sont sensiblement plus restreints que ceux du terme anglais. À quelques exceptions près, il n'y a pas de terme unique qui puisse être utilisé pour traduire pattern (le «mot manquant»). Par conséquent, les acceptions du terme anglais exigent des traductions différenciées. Le traducteur travaillant à partir du français doit reconnaître le mot pattern derrière les termes français, pour remettre ce «mot caché» là où il est requis dans la langue cible. L'article présente des cas d'espèce trouvés dans des sources françaises appariées pour le contexte.
\end{abstract}

\begin{abstract}
"Pattern" occurs in French as an anglicism; however, its meaning and scope of application are more restricted than those of the English term. With few exceptions, there is no one word that could be used as a French equivalent (the "missing word"). As a result, the meanings of "pattern" will need to be translated in other ways. The challenge to the translator working from French is to recognize "pattern" (the "hidden word") behind the French terms, and restore it as and where required in the target language. This paper gives equivalents of "pattern" found in context-matched French medical texts.
\end{abstract}

\section{MOTS-CLÉS/KEYWORDS}

pattern, hidden word, missing word, context-matching research

Attention: un mot peut en cacher un autre.

\section{Introduction}

Many centuries ago, the French word patron was borrowed by the English language. While, in French, the word retained its original sense (of "protector," designating a person, as well as of "model," designating an object), the borrowed word and its meaning developed along different lines. In the 16th century, the metathetical form "pattern" came to be applied to objects, while "patron" remained associated with persons. "Pattern" eventually acquired a large number of meanings more or less tenuously connected with the original sense of "model;" while "patron" had the meaning of "supporter" added to it. This, in turn, gave rise to a faux ami: in the phrase le patron et les consommateurs, the patron is the manager of the establishment, whereas the consommateurs would be the patrons in English.

Around 1920, "pattern" was borrowed back by the French language, where it remains in use as an Anglicism. The definition given in Le Dictionnaire de notre temps (1989) is typical of many others: pattern (anglicisme) En sciences humaines, modèle simplifié, schéma à valeur explicative, représentant la structure d'un phénomène

Meta, XLVI, 1, 2001 
complexe. (Équivalent fr.: modèle). Such terms as patron or schéma, schème have also been suggested as French equivalents.

A comparison of the definition and the scope of application of French pattern with the definitions of the English term in The Oxford English Dictionary (1989) shows the meanings and the uses of the French term to be very much more limited than those of its English counterpart. ${ }^{1}$

In medicine, the problem is not so much one of avoiding the use of an anglicism as of finding ways of rendering the many meanings of the English term in French. Suggested translations include aspect, profil, tableau, and tracé (Quérin 1998). The Gladstone (Gladstone 1996) gives a useful list of possible renderings. A recent Canadian publication (Leblanc 1999) contains a well-researched and meticulously referenced discussion of what it describes as a "terme caméléon."

The current study was undertaken to discover how the many meanings of English "pattern" may be rendered in French medical texts.

\section{Material and Methods}

The collection of examples upon which this paper is based was created for a course of medical English. ${ }^{2}$ In an exercise entitled "The Missing Word / Hidden Word," the point was made that a word in one language may not have a single, all-purpose equivalent in another language (the "missing word"). Students were then given the list of French examples, and asked to work out which English word could be used in the translation of all of the examples given (the "hidden word"). ${ }^{3}$ The terminology project is still going on.

The list was prepared by finding examples of the use of "pattern" in Englishlanguage material (textbooks, journals, oral communications by native authors), and matching them with French terms found in French material covering a similar context.

The list of French textbooks consulted included such series as the $A B C$ (e.g. Chirurgie plastique et esthétique) and the Abrégés (Radiodiagnostic, Sémiologie médicale, etc.) published by Masson; the Médecine Sciences Flammarion Cas cliniques (en dermatologie; en neurologie; etc.); and the ... pour le praticien range (e.g. Rhumatologie; Cardiologie) published by SIMEP. The Vademecum clinique du diagnostic au traitement by Fattorusso and Ritter (Paris, Masson; latest—15th-edition 1998) was a mine of information, as was Redécouvrir l'examen clinique. Clé du diagnostic (O. Blétry et al., 1995, Paris, Doin Éditeurs). Among the single-discipline texts were Pratique médico-légale (M. Durigon, 1999, Paris, Masson); Dermatologie et vénéréologie (J.-H. Saurat et al., 1991, 2nd ed., Paris, Masson); and Pneumo-clinique. Cas par cas (P. Camus (ed.), 1999, Paris, Margaux Orange). Two Histoire des hôpitaux titles (Accueillir et soigner-l'AP-HP, 150 ans d'histoire. F. Salaün (ed.), 1999, Paris, Doin Éditeurs / Assistance publique-Hôpitaux de Paris. Des femmes, des hommes, un hôpital. Le personnel de l'AP-HP témoigne. V. Rousset and Y. Spadoni (eds), 1999, Paris, Assistance Publique-Hôpitaux de Paris / Doin).

The periodical literature comprised Impact médecin and its supplements (Les dossiers du praticien; Les dossiers de FMC du praticien) 1989-1997; Maittrise orthopédique 1994- ; and the invaluable Revue du praticien (Paris) 1989-. For research in the (scientifically and terminologically) new field of developmental biology, La Recherche 
contained some useful material, while the last six years of Médecine Sciences proved a very important source.

Electronic sources were used much less than printed ones. In particular, MEDLINE was not resorted to. Some useful information came from such Internet sites as $<$ http://med.univ-rennes1.fr $>$ and $<$ http://anapath.necker.fr $>$.

The use of dictionaries was confined to the consultation of monolingual encyclopaedic texts.

Where required, oral information was obtained from French experts, to whom I am much indebted for their help and patience. In particular, I wish to thank the Institut Pasteur, Paris, for help with HIV epidemiological terminology; the Équipe INSERM 0012/Université Montpellier II, for advice on terminology in the field of developmental biology; to the Unité ULP-CNRS ULA 1295, Strasbourg, for assistance with French equivalents in ethology; and the Unité d'électromyographie et de pathologie neuro-musculaire of the Hôpital Pierre-Wertheimer, Lyon, for a detailed reply to my enquiry concerning interference patterns.

\section{Results}

For purposes of this paper, the original collection of equivalents has been rearranged in such a way as to reflect the meanings of the word "pattern." The definitions were derived from The Oxford English Dictionary (1989). Some of these definitions have been amplified. The different forms ("pattern," "patternless," "to pattern," "patterned," "patterning") are listed separately.

The very specific sense of "pattern(ing)" in developmental biology (a usage not yet covered by the $O E D$ ) is dealt with in a separate section.

In the examples given below, context is indicated, where required, between square brackets; French ways of rendering "pattern" are indicated by underscoring.

\section{pattern}

1. pattern = anything fashioned, shaped, or designed to serve as a model from which something is to be made; a model, design, plan, or outline.

[hospital patterns] des modèles pour les constructions hospitalières; les conceptions architecturales hospitalières; les hôpitaux conçus suivant les principes germaniques; des hôpitaux construits selon ce principe pavilion pattern / le système pavillonnaire; le concept pavillonnaire; l'architecture pavillonnaire; les projets pavillonnaires sont abandonnés

[nurses' caps] standard pattern / les bonnets blancs des infirmières seraient confectionnés d'après un modèle type

[block design test] - to replicate patterns using a set of blocks / l'enfant doit reproduire, avec des cubes, le dessin qui lui est proposé

[human form] le singe et la poupée ressemblent fortement au prototype "homme»

[ethology - Konrad Lorenz' Kindchenschema] baby pattern / schéma «bébé»

2. pattern $=$ a model or design $\ldots$ from which material $\ldots$ can be cut out [pattern drawn on skin prior to plastic surgery] dessin d'un patron sur les seins; l'ensemble de ces repères cutanés tracés sur le ventre

3. pattern $=$ a figure in wood or metal from which a mould is made for casting [hip prostheses] chacun apportait ses dessins, ses moules sur plâtre et autres bois sculptés 
4. pattern $=$ something formed after a model or prototype; a design; a model or version of a design; a type

[hip prostheses] - the Charnley pattern / la prothèse de Charnley

5. pattern $=$ a decorative or artistic design

[block design test] pattern on cubes / des cubes portant diverses dispositions colorées sur leurs six faces; un élément de figure géométrique; des figures colorées; reproduire des dessins géométriques

[visual evoked potentials] checkerboard pattern / damier

6. pattern = figuring or marking of natural or fortuitous origin

pattern $=$ an arrangement or order of things or activity in abstract senses

\subsection{ANATOMY}

normal anatomical pattern / anatomie normale vascular pattern / vascularisation a rich vascular pattern / une grande richesse vasculaire iliac crest crossing pattern [nerves] / les modalités de croisement de la crête iliaque distinctive pattern [pulmonary veins on chest X-ray] / les veines pulmonaires n'ont pas une individualité suffisante pour être dissociées du réseau artériel

dermal ridge pattern; pattern of ridges on the skin / disposition des lignes papillaires; les figures formées par les crêtes papillaires; dermatoglyphes trabecular pattern / trabéculation mucosal pattern / relief muqueux normal mucosal pattern / le plissement muqueux normal standard vertebral pattern / la vertèbre fondamentale

knee pattern / la conformation du genou condylar pattern / chaque genou montre un dessin de profil condylien qui lui est propre

pattern of the tibia [e.g. tibia vara, tibia valga] / morphotype du tibia

[foot] digital patterns / les principaux canons d'orteils

[skin lesion; shadow on X-ray] based on an anatomical pattern / systématisé

\subsection{PHYSIOLOGY AND PATHOPHYSIOLOGY}

sleep pattern / la structure du sommeil; l'organisation du sommeil; les phases du sommeil disturbed sleep pattern / perturbation du déroulement $d u$ sommeil; sommeil perturbé [blood flow] laminar flow pattern - turbulent flow pattern / l'écoulement laminaire fait place à un régime turbulent

[reflexes] neonatal reflex patterns / réflexes archaïques du nouveau-né; réflexes primaires $d u$ nouveau-né

pain pattern / on cherche à rapprocher la douleur d'un modèle connu; moduler les prises d'antalgique en fonction de l'algorithme de la douleur dans les 24 heures muscle patterns / des schèmes musculaires

physiological stress pattern / le respect de la carte des contraintes naturelles gait pattern / démarche; la marche est perturbée respiratory pattern / rythme respiratoire

[respiratory distress] see-saw pattern of respiration / balancement thoraco-abdominal hormone pattern / le profil hormonal

secretory pattern / rythme de sécrétion; la rythmicité des sécrétions; profil de sécrétion; algorithme de sécrétion; sécrétion algorithmique circadian pattern / rythme nycthéméral; rythme circadien; profil d'évolution au cours des 24 heures; la température centrale suit une évolution circadienne; le prurit est rythmé par le nycthémère

\subsection{EPIDEMIOLOGY}

epidemiological pattern / profil épidémiologique; les modalités épidémiologiques; la situation épidémiologique; type épidémiologique; l'épidémiologie au Maghreb rejoint celle des pays développés; l'épidémiologie bactérienne des services de réanimation 
changes in the epidemiological pattern / les modifications épidémiologiques

pattern of spread [of a disease] / modalité épidémiologique; mode de transmission; modalités de dissémination; allure épidémiologique; diffusion; dissémination

geographical pattern / un facteur géographique; la géographie de la maladie

disease with a seasonal pattern / maladie à caractère saisonnier

no seasonal pattern / survenue en toute saison

no ethnic pattern / absence d'électivité ethnique; pas de prédilection ethnique

age pattern / distribution selon les classes d'âge; distribution en fonction des classes d'âge regional mortality patterns / répartition régionale de la mortalité générale

distinct epidemiological patterns / situations épidémiologiques distinctes

[HIV infection - terminology used in the early 1990s] Pattern I / répartition de type I

[HIV infection - terminology used in the early 1990s] Pattern II / répartition de type II

[HIV infection - terminology used in the early 1990s] Pattern II country / pays de type II;

pays "pattern II;" pays où la transmission est hétérosexuelle

\subsection{PATHOLOGY AND CLINICAL MEDICINE}

abnormal pattern / les éléments pathologiques

pathological pattern / anatomie pathologique

the pathological pattern is ... / les lésions histologiques sont caractérisées par ...

fracture patterns / les types de fracture

injury pattern / la formule lésionnelle; la topographie des blessures

clinical pattern(s) / les tableaux cliniques; les formes cliniques; les anomalies chromosomiques

donnent des tableaux variés; types cliniques; situations cliniques; l'apparence clinique

disease pattern / le visage de la maladie; la physionomie de la maladie; le caractère d'une

céphalée; le masque habituel d'une maladie; la forme de la maladie en Europe

pattern of obesity / la forme topographique de l'obésité male pattern of fat distribution / obésité androïde

male-pattern baldness; male-pattern alopecia / alopécie androgénétique; calvitie de type

masculin

[hirsutism] male pattern of hair distribution / hypertrichose à distribution masculine

fever pattern; febrile temperature pattern; pattern of fluctuation on the temperature chart /

type de fièvre; type de courbe thermique; l'allure de la courbe thermique; le profil d'une fièvre

[fractures] the healing pattern is influenced by age/ le temps de consolidation peut varier selon

l'âge du blessé

typical footprint patterns / types d'empreinte plantaire; types d'assise plantaire

[caput medusae] a pattern of dilated cutaneous veins radiating from the umbilical area / un lacis veineux péri-ombilical

lipoprotein patterns [= specific types of hyperlipoproteinaemia] / types d'hyperlipoprotéinémie; hyperlipoprotéinémies

[familial combined hyperlipidaemia] different lipoprotein patterns in different affected members / présence dans une même famille de phénotypes lipoprotéiniques variables [type III hyperlipoproteinaemia] a pattern known as apo $\mathrm{E}_{2} / \mathrm{E}_{2}$ is diagnostic / le phénotype E2E2 dont la découverte devient un argument diagnostique

[acute nephritic syndrome] immune complex deposition with IgG and C in a granular pattern / dépôts granuleux d'IgG et de la fraction C3 du complément

[malignant mesothelioma] a wide variety of morphological patterns; different growth patterns; three patterns / trois variétés anatomo-pathologiques; trois types histologiques sont décrits biphasic pattern / forme mixte; mésothéliome mixte; mésothéliome biphasique [different patterns] trois ordres de fait ont pu être constatés (densification, calcification, absence de liseré); [many different patterns] les anomalies cardiovasculaires congénitales sont d'un polymorphisme extrême; [unvarying pattern] un monomorphisme clinique; la bactériologie est très monomorphe (trois germes régulièrement impliqués); la maladie peut paraître stéréotypée; [monotonous pattern] une population cellulaire d'aspect monotone [histology of Paget's disease] mosaic pattern / structure irrégulière "en mosaïque" jigsaw pattern / désorganisation des travées osseuses qui sont juxtaposées comme les pièces d'un puzzle 
6.5. INVESTIGATIONS

- ELECTROPHORESIS

electrophoretic pattern / aspect électrophorétique; tracé électrophorétique

- IMMUNOHISTOCHEMISTRY

immunostaining pattern / les adénomes de la thyrö̈de ont un immunomarquage

à la thyropéroxydase positif

- DNA ANALYsis

banding pattern / une succession de bandes

genetic pattern; DNA pattern / empreinte génétique pattern unique to each individual /

configuration particulière des séquences d'ADN d'un individu donné

- EEG

EEG sleep patterns / les images Eeg du sommeil

abnormal EEG patterns; abnormal wave patterns / des anomalies EEG

- ECG

ECG patterns; patterns on the ECG / les modifications de l'activité électrique se traduisent par des tracés caractéristiques; signes électriques; aspects ECG

[myocardial infarction - ST segment elevation] injury pattern / image de lésion; signes

électrocardiographiques de lésion; courant de lésion décalant le segment ST au-dessus de la ligne isoélectrique

"early repolarization" pattern / repolarisation précoce

- EMG

interference pattern [ = motor unit recruitment pattern where baseline is obscured and individual potentials are no longer distinguishable]; full interference pattern; complete interference pattern / tracé EMG (d'effort) interférentiel; tracé EMG riche; état d'interférence full interference pattern at less than maximal contraction / tracé intermédiaire riche; tracé interférentiel d'emblée; tracé interférentiel précoce interference pattern more complete than normal / tracé intermédiaire trop riche; tracé trop riche pour la force déployée; tracé interférentiel d'emblée; recrutement excessif pour la force interference pattern [= motor unit recruitment pattern in general] / recrutement des unités motrices

normal interference pattern / recrutement normal des unités motrices

reduced interference pattern; incomplete interference pattern / tracé EMG appauvri; tracé pauvre; tracé intermédiaire; recrutement appauvri; appauvrissement du tracé en unités motrices discrete interference pattern; single-unit interference pattern; picket-fence interference pattern I tracé simple; tracé simple accéléré; tracé très pauvre

- EVOKED POTENTIALS

[visual evoked potentials] pattern reversal visual evoked potentials / [potentiels évoqués visuels] potentiels évoqués par l'inversion d'un damier

- LUNG FUNCTION TESTS

a restrictive pattern / trouble ventilatoire restrictif; insuffisance du type restrictif; les EFR sont $\underline{\text { restrictives }}$

mixed obstructive-restrictive pattern / trouble ventilatoire mixte

- IMAGING TECHNIQUES

angiographic patterns / images angiographiques

scintigraphic pattern / signature scintigraphique

ultrasound patterns; sonographic patterns / la sémiologie échographique des veines normales; la sémiologie ultrasonore; l'échostructure d'une lésion; les calculs vésiculaires ont une traduction acoustique uniforme; l'aspect évocateur échotomographique du calcul; l'imagerie chronologique de la grossesse

radiographic pattern(s) / la sémiologie radiologique; signes radiologiques; aspect radiologique; expression radiologique; l'image de base spécifique de l'ostéonécrose; la structure radiologique normale du poumon; une radiologie caractéristique [abdominal plain film] abdominal gas pattern / la répartition de l'air sur les clichés d'abdomen sans préparation

CT pattern(s) / la séméiologie tomodensitométrique; l'aspect tomodensitométrique 
[MRI - enhancement with gadolinium] enhancement pattern; pattern of enhancement / type de prise de contraste [gallstones] triradiate gas pattern / «étoile gazeuse» [osteosarcoma] sunburst pattern / aspect «en feu d'herbes»; image «en feu d'herbes» [sickle-cell anaemia] hair-on-end pattern / crâne en poils de brosse; image en poils de brosse [multiple myeloma] moth-eaten pattern / lacunes à l'emporte-pièce; aspect à l'emporte-pièce [chest X-ray in emphysema] attenuation of vascular pattern / raréfaction vasculaire diffuse [chest X-ray in interstitial lung disease] honeycomb pattern / image en nid d'abeille; aspect en "nid d'abeilles»; image en rayon de miel; aspect en rayon de miel; poumon en rayon de miel; rayon de miel [as heading in table; or as in le rayon de miel très discret] ground-glass pattern / aspect en verre dépoli; images en verre dépoli; le verre dépoli mosaic pattern / mosaïque

patterns that mimic interstitial lung disease / faux syndromes interstitiels [chest X-ray in pulmonary oedema] butterfly pattern; bat's wing pattern / image radiologique en ailes de papillon

[vertebra in secondary hyperparathyroidism] rugger-jersey pattern / vertèbre en sandwich [asbestos-related diseases] the X-ray pattern will have changed before the spirometry one / les anomalies radiologiques sont en général plus précoces que les anomalies fonctionnelles [high resolution computed tomography] HRCT enhances pattern recognition / la tomodensitométrie haute résolution est plus sensible pour dépister et préciser les lésions; la TDMHR permet une étude plus précise; la TDM-HR met en évidence des images mieux définies - BIOCHEMistry amino acid pattern / aminoacidogramme protein pattern / un lait avec des concentrations protéiques un peu différentes de celles du lait maternel

\subsection{DERMATOLOGY}

shape and pattern of lesions / la forme et l'arrangement des lésions élémentaires entre elles; forme et configuration des lésions; la nature et le groupement des lésions élémentaires linear, annular, grouped patterns / disposition linéaire, disposition annulaire, lésions groupées a nodular pattern / un agencement nodulaire bizarre pattern / l'aspect bizarre des lésions an easily recognizable pattern / une topographie évocatrice; une disposition très caractéristique [atopic eczema] reverse pattern / inversion des localisations

[atopic eczema] the pattern varies with age / le tableau clinique est variable selon l'âge; les manifestations cliniques varient dans leur nature et leur distribution selon l'âge du patient cutaneous reaction patterns / lésions élémentaires histologiques de la peau zosteriform pattern; dermatomal pattern / groupement zostériforme; topographie zoniforme; topographie métamérique dessinant le territoire d'une racine sensitive; lésions orientées selon les dermatomes net-like pattern; reticulate pattern / réseau; $\underline{\text { arborisations }}$ [lichen planus] lesions on the insides of the cheeks arranged in a network pattern of thin thread-like streaks / [atteinte buccale] lésions blanchâtres en réseau (réseau en feuilles de fougère) [poikiloderma] speckled pattern / aspect bigarré de l'épiderme; réseau bigarré [pityriasis rosea] Christmas-tree pattern; Christmas-tree-like pattern / dessin en sapin de Noël [Ehlers-Danlos syndrome] prominent venous pattern / visibilité anormale du réseau veineux sous-dermique; la peau fine, transparente laisse voir le réseau vasculaire [incontinentia pigmenti] patterns of striae and whorls / hyperpigmentation en tourbillons, en jet d'eau, en feu d'artifice whorled pattern / disposition tourbillonnante hyperpigmentation in the pattern of Blaschko's lines / hyperpigmentation figurée suivant le tracé des lignes de Blaschko pattern distributed along the lines of Blaschko / lésions orientées selon les lignes de Blaschko pattern of spread [of skin lesions] / topographie et évolution dans le temps de l'exanthème; mode d'extension; déroulement de l'exanthème; l'acné débute au front, puis gagne le bas du visage et le dos et la poitrine. La résolution suit le même parcours, le visage guérissant en premier. 
6.7. GYNAECOLOGY

[fern test for ovulation] fern frond pattern; frondlike pattern / cristallisation de la glaire cervicale en feuille de fougère

[colposcopy] coarse punctate pattern / semis de gros points rouges; pointillé rouge biphasic pattern of the basal body temperature curve / la courbe thermique normale biphasique; le tracé de la courbe thermique comporte normalement deux phases

\subsection{SURGERY}

[plastic surgery, flaps] axial-pattern flaps - random-pattern flaps / lambeaux cutanés axiaux lambeaux cutanés au hasard

[minimal access therapy] pattern of trocar placement / position des trocarts; positionnement des trocarts; placement des trocarts; emplacement des trocarts

\subsection{FORENSIC MEDICINE}

[pressure abrasion retaining an imprint of the causative object] pattern left on the skin / [abrasion par pression] le parcheminement secondaire montre un aspect en forme correspondant à l'agent vulnérant fingerprint patterns [arches, loops, whorls] / figures pulpaires [arches, boucles, tourbillons] fingernail ridge patterns / dessin des stries longitudinales; $\underline{\text { stries longitudinales }}$

7. pattern $=$ order or form discernible in things, actions, ideas, situations, etc.

\subsection{BEHAVIOUR}

behaviour patterns / comportements; conduites; traits comportementaux; styles de comportement; types de comportement eating patterns; feeding patterns / les habitudes alimentaires; l'existence de comportements boulimiques; une manipulation inhabituelle des aliments [anorexie mentale] abnormal behaviour patterns / des conduites pathologiques regular pattern / créer un automatisme [conditions d'un sommeil régulier]; rompre l'automatisme; un autre risque pour le diabétique, la rupture de la vie linéaire menée tout au long de l'année

[risk factor for myocardial infarction] Type A pattern of behaviour / schéma de comportement de type A; comportement de type A

[ethology] fixed action pattern / séquence stéréotypée d'actes instinctifs

[ethology] innate release pattern / patron inné de déclenchement

\subsection{GENETICS}

pattern of inheritance; inheritance pattern / mode de transmission génétique; modalité de transmission génétique; type de transmission; forme de transmission; transmission [as in: la maladie est de transmission liée au chromosome $X]$

hair loss follows a family pattern / la distribution de l'alopécie a un caractère familial a family pattern [= a family history] / une histoire familiale; des antécédents familiaux familial pattern / forme(s) familiale(s); incidence familiale élevée

\subsection{MOVEMENT}

[hospital planning] patterns of circulation / un organigramme de circulations différenciées [staff time \& motion] movement patterns / trajets

\subsection{TIME COURSE}

circadian pattern of blood theophylline levels / cycles nycthéméraux de la théophyllinémie time pattern / une périodicité de 7 jours; sa dépression, cyclique, obéissait à un scénario bien rodé

\subsection{SITUATION}

[hausse du nombre de médecins] on retrouve des schémas équivalents dans 7 autres départements; au regard de cette évolution

[fécondité] on retrouve quatre cas de figure bien différents lorsqu'on parle des pays en développement 
[sex ratio et âge des malades] ces données sont aussi constatées par...

[résection du coude] les muscles sont très bien adaptés à ce nouvel état de choses [complex pattern] tout un jeu complexes d'interactions; des interactions dont la trame est mystérieuse

\subsection{ORDER IN THINGS; ORDERLY ARRANGEMENT}

l'apparition spontanée d'un ordre; une constante se dégage; une «logique»; la belle ordonnance d'un réseau d'atomes de carbone; la courbe thermique n'affecte pas toujours la belle régularité classique

8. pattern $=$ the marks made by the shot from a gun on a target, in respect of their closeness together and evenness of distribution within a certain radius from the central point [forensic medicine] pattern of a shotgun / groupement des plombs d'un fusil de chasse; regroupement des plombs

9. pattern = in Ireland, a patron saint's day; hence the festivities with which it is celebrated le pardon de Notre-Dame-de-Toute-Aide permit à une petite sourde-muette de retrouver l'oü̈e et la parole.

\section{patternless}

= conforming to, or possessing, no discernible arrangement or pattern [polio] the spread of the paralysis shows no consistent pattern / les paralysies apparaissent de façon anarchique et asymétrique [Paget's disease] patternless trabeculae; trabeculae developing in a disorganized, patternless manner / structure irrégulière, anarchique; remodelage osseux anarchique; caractère anarchique $d u$ remodelage osseux; disposition irrégulière des travées osseuses [atrial fibrillation] chaotic pattern / rythme totalement irrégulier; irrégularité du rythme; désorganisation de l'activité électrique; l'activité auriculaire est désordonnée; activité irrégulière et imprévisible

no discernible pattern / n'obéit à aucune systématisation; la typologie est mise à défaut

\section{to pattern}

[Doman \& Delacato's technique for the treatment of children with cerebral palsy] to pattern a child / enseigner des programmes neuromoteurs; faire de la programmation neuromotrice; automatiser des programmes neuromoteurs

to pattern something on something = to make something after a model: une initiative calquée sur le système de transfusion sanguine; les carrières des personnels ouvriers de l'Assistance Publique s'inspirent étroitement de celles des agents de la ville de Paris

to pattern oneself on someone $=$ to take someone as a model, as an exemplar: Ambroise Paré, prenant exemple sur Galien...

\section{patterned}

= decorated with a pattern or design

[block design test] nine patterned cubes / des cubes portant diverses dispositions colorées sur leurs six faces; les cubes portent sur chaque face un élément de figure géométrique

= having a pattern; conforming with, or forming, an arrangement or pattern [lichen planus] geometrically patterned lesions / des lésions élémentaires à contours polygonaux; caractère polygonal des lésions [piebaldism] a patterned leukoderma / se caractérise par des macules achromiques [naevoid hypermelanosis] a patterned hyperpigmentation / une hyperpigmentation figurée [forensic medicine] patterned abrasion / abrasion «en forme" p patterned print / empreinte «en forme» 


\section{patterning}

$=$ pattern

[dermatology] the linear patterning follows Blaschko's lines / lésions linéaires orientées selon les lignes de Blaschko; dermatose linéaire disposée selon les lignes de Blaschko

[Doman \& Delacato's technique for the treatment of children with cerebral palsy] patterning; méthode de Doman-Delacato

\section{“pattern(ing)" in developmental biology}

In developmental biology, "pattern formation" is defined (Thain \& Hickman 2000) as "... phenomena whereby cells in different parts of embryo become locked into different spatio-temporal developmental pathways, coordinated in such a way as to produce a viable multicellular system."

body pattern [= body plan] / plan (général) du corps; plan de construction; plan d'organisation (corporelle); schéma corporel; plan d'ensemble; plan de développement

pattern-specifying gene; pattern formation gene; patterning gene / gène organisateur; gène réglant la morphogenèse; gène qui intervient dans la morphogenèse; gène qui détermine la formation de ...; gène qui détermine la genèse de ...; gène impliqué dans la construction de ...; gène régissant l'organisation de ...; gène responsable de la formation de ...

to determine the pattern of ... / déterminer l'identité de ...

pattern duplications / duplications de structures; formation des structures surnuméraires abnormal patterns / anomalies

pattern of expression; expression pattern / profil d'expression

prepattern; prepatterning / organisation préalable; pré-organisation; pré-motif

to pattern a structure / organiser; structurer; déterminer l'identité de ...; conférer une identité à ...

to pattern / agir en tant que morphogène

patterning of the body / l'établissement de l'organisation; définition du plan du corps; formation des structures; élaboration du schéma corporel; constitution de la morphologie

fundamental patterning / schéma de base

limb patterning / organisation du membre; morphogenèse du bourgeon de membre; organisation spatiale du bourgeon de membre

patterning of the final structure / mise en place de l'architecture du futur adulte; constitution de la topographie du futur embryon

dorsoventral patterning / organisation dorso-ventrale; détermination du plan d'organisation le

long de l'axe dorso-ventral

to control dorsoventral patterning / déterminer la polarité dorso-ventrale; agir sur la polarisation dorso-ventrale

anterior-posterior patterning / l'organisation sur l'axe $A / P$

long-range patterning / action à distance d'un morphogène

patterning information / information organisatrice; information structurante

early patterning events / les premières étapes de l'émbryogenèse

développement précoce

patterning function / activité organisatrice; influence organisatrice; propriétés organisatrices prepatterning genes / gènes de pré-motif; gènes de pré-organisation

\section{Discussion}

As may be seen from the results reported above, there are many different ways of rendering English "pattern" in French. The list provided in this paper does not claim to be exhaustive (some subject areas are, in fact, under-represented), and is still being added to.

Two important French patterns to emerge from this study are the frequent use of such "all-purpose" terms as aspect, type, disposition; and the use of phrases involving en (image en..., vertèbre en... etc.). 
The use of the Anglicism pattern does not appear to be a problem in French medical texts. In fact, as the examples from the field of developmental biology show, French writers have found many different ways of expressing the meanings of the English term.

The fact that the English term "pattern" or a particular French translation have been listed under Results should not be interpreted to mean that "pattern" would be universally used in that context, or that the French translation given is the only possible one. (It should also be borne in mind that, as pointed out by L. Leblanc (1999), "le recours aux listes de correspondants est d'une utilité limitée: la traduction de ce terme impose plutôt une recherche et une analyse rigoureuse.")

The terms used in the English definitions show the range of less general expressions that may be used; also, "trabecular pattern" may be replaced by "trabeculation," and such terms as "arrangement," "configuration," or "distribution" are widely used in a variety of texts. Some authors appear to be averse to the use of "pattern," and prefer these alternative terms. However, even those who do use "pattern" will often employ the alternatives, to avoid repetition or for the sake of brevity when referring back to earlier, more detailed statements (e.g. [narcolepsy] "Onset of REM sleep is almost instantaneous. This pattern differs from normal sleep, in which NREM sleep usually lasts about 60 to $90 \mathrm{~min}$, preceding REM sleep." / "L'électroencéphalogramme montre dès l'endormissement, ou peu après, une période de sommeil paradoxal qui, chez le sujet normal, ne survient qu'après une certaine durée d'endormissement de l'ordre de 90 minutes environ.").

In general, "pattern" appears to be more widely used in recent, as compared with older, texts. The recent Centennial (17th) edition of The Merck Manual (Beers \& Berkow 1999) contains many examples of the use of the term. (I am grateful to Dr. Berkow for photocopies of a large number of passages containing "pattern", and to his co-editor for drawing my attention to the availability of the manual on the Internet.) While some would agree with Quérin (1998) that the term is being used to excess in the English-language literature, "pattern" is useful, for a number of reasons. (1) It allows a distinction to be made between the paradigm and the specific case: a "pedigree pattern" is the arbre généalogique-type, e.g. of an autosomal dominant disorder; the individual patient's arbre généalogique would be his or her "pedigree;" similarly, "pedigree pattern" may be used for the drawing representing the patient's pedigree ("construction of a pedigree pattern"). (2) It allows the pluralization of words that are not normally used in the plural: "behaviour patterns" (rather than the distinctly rare "behaviours") as the English rendering of French comportements, conduites is a case in point. (3) It allows greater economy of expression: "les manifestations cliniques varient dans leur nature et leur distribution selon l'âge du patient;" or "l'écartement des blessures et la topographie" may be, succinctly, translated as "the pattern varies with age," and "the injury pattern," respectively. (4) The term is very English-witness the problems encountered when it needs translating into other languages. Its use in a translation would achieve what I consider to be the hallmark of a good translation: a text that is sufficiently far removed from the source language to make it difficult to translate back into that language.

Context-matching research has an additional benefit: it acts as a sort of pattern drill or pattern practice (exercice de structure), enhancing the researcher's skill of pattern recognition (reconnaissance des formes). In a reversal of the approach de- 
scribed above, I have, of late, identified French terms behind which "pattern" may be hiding, and checked out my hunch against context-matched English sources. Thus, the French term coulures, in the description of blood stains in a forensic medicine textbook, made me suspect that a "pattern"-term might be used in English. This guess was confirmed by a television programme on forensic medicine, where one of the experts used the term "running pattern."

In conclusion, finding the ways in which the French language makes up for the fact that, in the vast majority of cases, there is no one equivalent of "pattern" is one of the most intriguing aspects of linguistic research. Similarly, using "pattern" as the translation of the terms thus found is one of the more rewarding aspects of the translator's craft.

\section{NOTES}

1. The problem of how to translate "pattern" exists also in German. While German Muster shares several of the meanings of "pattern," it tends to be overused. Terms such as Verlauf, Erscheinung(sform), Art, Schema, Ablauf, Typ, Verhältnis(se), Konfiguration, Gesetzmässigkeit, etc. would often be a better choice.

2. CMETI, the Course of Medical English for Translators and Interpreters, was an annual two-week event, at a London teaching hospital, from 1987 to 1997.

3. Similar exercises were run, from English into French, concerning renderings of the word "control;" and from French into English, concerning such terms as malaise; lourd / léger; and le moignon de l'épaule.

\section{REFERENCES}

BeErs, M.H. and R. Berkow (eds) (1999): The Merck Manual of Diagnosis and Therapy, Whitehouse Station (N.J.), Merck \& Co., Inc. Available on the Internet at $<$ http://merckmanual.com $>$.

Gatard, M. (dir.) (1989): Le dictionnaire de notre temps, Paris, Hachette, p. 1120.

Gladstone, W.J. (1996): Dictionnaire anglais-français des sciences médicales et paramédicales / English-French Dictionary of Medical and Paramedical Sciences, 4th ed., Saint-Hyacinthe, Edisem; Paris, Maloine.

Leblanc, L. (1999): «Dégagement du ciel sémantique de pattern: une tâche ponctuelle et contextuelle», Pharmaterm. Bulletin terminologique de l'industrie pharmaceutique (Groupe traduction $\mathrm{Rx} \&$ D.), 10-4.

Oxford English Dictionary, The (1989), 2nd ed., Oxford, Clarendon Press, pp. 356-359

QuÉRIN, S. (1998): Dictionnaire des difficultés du français médical, Saint-Hyacinthe, Edisem; Paris, Maloine, p. 133.

Thain, M. and M. Hickman (2000): The Penguin Dictionary of Biology, $10^{\text {th }}$ ed., London, Penguin Books, p. 479. 